\title{
EXACT COHOMOLOGY SEQUENCES WITH INTEGRAL COEFFICIENTS FOR TORUS ACTIONS
}

\author{
MATTHIAS FRANZ AND VOLKER PUPPE
}

\begin{abstract}
Using methods applied by Atiyah in equivariant $K$-theory, Bredon obtained exact sequences for the relative cohomologies (with rational coefficients) of the equivariant skeletons of (sufficiently nice) $T$-spaces $X, T=\left(S^{1}\right)^{n}$, with free equivariant cohomology $H_{T}^{*}(X ; \mathbb{Q})$ over $H_{T}^{*}(\mathrm{pt} ; \mathbb{Q})=H^{*}(B T ; \mathbb{Q})$. Here we characterise those finite $T$-CW complexes with connected isotropy groups for which an analogous result holds with integral coefficients.
\end{abstract}

\section{INTRODUCTION}

Let $T=\left(S^{1}\right)^{n}$ be an $n$-dimensional torus. By a $T$-space we mean a finite $T$ CW complex, for example a smooth compact $T$-manifold. $H^{*}(-)$ denotes singular cohomology with integral coefficients unless stated otherwise. Let $X_{i},-1 \leq i \leq n$, be the equivariant $i$-skeleton of $X$, i. e., the union of all orbits of dimension $\leq i$. In particular, $X_{-1}=\emptyset, X_{0}=X^{T}$ and $X_{n}=X$.

Recall that the $T$-equivariant cohomology $H_{T}^{*}(X)$ of $X$ is defined as the (singular) cohomology of the Borel construction $X_{T}=(E T \times X) / T$ and that $H_{T}^{*}(X)$ is a module over $H^{*}(B T)$, where $E T \rightarrow B T$ denotes the universal $T$-bundle. We also consider $\mathbb{Z}$ as an $H^{*}(B T)$-module by the augmentation $\varepsilon: H^{*}(B T) \rightarrow \mathbb{Z}$ that annihilates all elements of positive degrees.

The inclusion of pairs $\left(X_{i}, X_{i-1}\right) \hookrightarrow\left(X, X_{i-1}\right)$ gives rise to a long exact sequence in equivariant cohomology

(1.1) $\cdots \rightarrow H_{T}^{*}\left(X, X_{i}\right) \rightarrow H_{T}^{*}\left(X, X_{i-1}\right) \rightarrow H_{T}^{*}\left(X_{i}, X_{i-1}\right) \rightarrow H_{T}^{*+1}\left(X, X_{i}\right) \rightarrow \cdots$, and likewise $\left(X_{i+1}, X_{i-1}\right) \hookrightarrow\left(X_{i+1}, X_{i}\right)$ gives boundary maps

$$
H_{T}^{*}\left(X_{i}, X_{i-1}\right) \rightarrow H_{T}^{*+1}\left(X_{i+1}, X_{i}\right) .
$$

Theorem 1.1. Let $X$ be a T-space. Then the following four conditions are equivalent:

(i) The inclusion of the fibre $\iota: X \hookrightarrow X_{T}$ induces a surjection $\iota^{*}: H_{T}^{*}(X) \rightarrow$ $H^{*}(X)$. Equivalently, the map $H_{T}^{*}(X) \otimes_{H^{*}(B T)} \mathbb{Z} \rightarrow H^{*}(X)$ induced by $\iota^{*}$ is an isomorphism.

(ii) The Serre spectral sequence of the fibration $X \hookrightarrow X_{T} \rightarrow B T$ degenerates at the $E_{2}$ level, i.e., $E_{2}=E_{\infty}$.

(iii) $\operatorname{Tor}_{j}^{H^{*}(B T)}\left(H_{T}^{*}(X), \mathbb{Z}\right)=0$ for all $j>0$.

(iv) $\operatorname{Tor}_{1}^{H^{*}(B T)}\left(H_{T}^{*}(X), \mathbb{Z}\right)=0$.

The above conditions are implied by the next one.

(v) The following sequence is exact:

$$
0 \rightarrow H_{T}^{*}(X) \rightarrow H_{T}^{*}\left(X_{0}\right) \rightarrow H_{T}^{*+1}\left(X_{1}, X_{0}\right) \rightarrow \cdots \rightarrow H_{T}^{*+n}\left(X_{n}, X_{n-1}\right) \rightarrow 0 .
$$

Moreover, if all isotropy groups of $X$ are connected, then this last condition is equivalent to the others. 
The main contribution of this paper is to relate the criteria (ii)-(iv) to the exact sequence given in (V). For field coefficients, the equivalence of (ii) to (iv) is standard.

It is easy to see that for coefficients in a field $k$ each of the conditions (ii) to (iv) above is equivalent to $H_{T}^{*}(X ; k)$ being a free $H^{*}(B T ; k)$-module. This does not hold for integer coefficients, as Example 5.2 below shows.

The connectedness assumption on the isotropy groups will be used in Proposition 3.2 and Lemma 3.4 and cannot be omitted in general. But, as shown in [FP], one can allow one (finite) cyclic factor in each isotropy group if $H_{T}^{*}(X)$ is free over $H^{*}(B T)$. In Section 5.2 we present some examples of what can go wrong if these conditions are not satisfied. Moreover, one can obtain a partial result if the isotropy groups are connected for all $x$ in some $X_{i}$ and not "too much" disconnected for $x \notin X_{i}$, see Remark 4.2

That the freeness of $H^{*}(X ; \mathbb{Q})$ implies the exact sequence (V) (with rational coefficients) was pointed out by Bredon $[\mathrm{B}$, based on an analogous result in equivariant $K$-theory due to Atiyah [A, Lecture 7]. We therefore refer to sequence (V) as the "Atiyah-Bredon sequence".

Atiyah's proof relies on the theory of Cohen-Macaulay modules. In FP the same method is used to give a version for other coefficients, which also allows for non-connected isotropy groups under appropriate assumptions (see the discussion preceding Example 5.4 below). In the present paper, we instead apply fairly standard homological algebra which makes essential use of the grading and gives a more complete picture in the case of connected isotropy groups and integer coefficients. This approach was inspired by Barthel-Brasselet-Fieseler-Kaup, who prove a result analogous to the rational version of Theorem 1.1] in the context of (intersection) cohomology for fans BBFK, Theorem 4.3].

For $H_{T}^{*}(X ; \mathbb{Q})$ a free $H^{*}(B T ; \mathbb{Q})$-module, the exactness of the Atiyah-Bredon sequence at $H_{T}^{*}(X ; \mathbb{Q})$ is an immediate consequence of the Localisation Theorem, and the exactness at $H_{T}^{*}\left(X_{0} ; \mathbb{Q}\right)$ follows directly from the so-called Chang-Skjelbred lemma CS Lemma 2.3 \& Proposition 2.4]. Goresky-Kottwitz-MacPherson GKM] and others made very effective use of the Chang-Skjelbred result in calculating the (equivariant) cohomology of certain $T$-spaces, sometimes even with coefficients in $\mathbb{Z}$.

Remark 1.2. The proof for Theorem 1.1 given below simplifies if one takes rational (or real) coefficients instead of $\mathbb{Z}$. In this case the assumption about the connectedness of the isotropy groups can be dropped completely. The reason is that for any isotropy group $T_{x}$ one has that $H^{*}\left(B T_{x} ; \mathbb{Q}\right)$ is naturally isomorphic to $H^{*}\left(B T_{x}^{0} ; \mathbb{Q}\right)$ where $T_{x}^{0} \subset T_{x}$ denotes the identity component.

Remark 1.3. As with the Atiyah-Bredon method (cf. B]), our arguments can also be used to prove similar results for $p$-tori and coefficients in $\mathbb{F}_{p}$. Again, the proof simplifies since we can take as extension of $\mathbb{F}_{p}$ any infinite field of characteristic $p$ (e. g., the algebraic closure $\overline{\mathbb{F}}_{p}$ ) instead of the less familiar extension of $\mathbb{Z}$ introduced in Section [3] We nevertheless concentrate on the (compact) torus case and integer coefficients since we get new results in this context. Variants for compact tori and coefficients in $\mathbb{F}_{p}$ or in a subring of $\mathbb{Q}$ can be found in $[\mathrm{FP}$.

Remark 1.4. The assumption that $X$ is a finite $T$-CW complex could be weakened if one is willing to apply more technical machinery along the lines of $\mathrm{AP}$, Chapter 3].

Acknowledgements. The authors thank Walter Baur, Karl-Heinz Fieseler and Sue Tolman for helpful discussions, and the referees for their comments and suggestions.

\section{Some homological Algebra}

In this section we collect a few more or less known results from homological algebra for later use. 
We work over an arbitrary ground ring (commutative, with unit) $k$. Unless stated otherwise, tensor products and direct sums are taken over $k$. We set $A=k\left[t_{1}, \ldots, t_{r}\right]$ where each $t_{i}$ has degree 2 , and we call elements in $A^{2}$ (= the $k$-module generated by $\left.t_{1}, \ldots, t_{r}\right)$ linear. All complexes and (graded) modules are assumed to be bounded from below.

The following standard fact is immediate from the long exact Tor sequence.

Lemma 2.1. Let $0 \rightarrow L \rightarrow M \rightarrow N \rightarrow 0$ be a short exact sequence of $A$-modules such that $\operatorname{Tor}_{j}^{A}(M, k)=0$ for all $j>i$. Then $\operatorname{Tor}_{j+1}^{A}(N, k)=\operatorname{Tor}_{j}^{A}(L, k)$ for all $j>i$.

An important role in this section is played by the short exact sequence of $A[t]$ modules

$$
0 \rightarrow k[t] \stackrel{\cdot t}{\longrightarrow} k[t] \rightarrow k \rightarrow 0 .
$$

Lemma 2.2. Let $M$ be an $A[t]$-module (where $t$ is an indeterminate of degree 2 ), considered as an $A$-module by restriction, $A \hookrightarrow A[t]$. Then $\operatorname{Tor}_{1}^{A}(M, k)$ vanishes if one of the following conditions holds:

(i) $\operatorname{Tor}_{1}^{A[t]}(M, k)=0$,

(ii) $\operatorname{Tor}_{2}^{A[t]}(M, k)=0$ and $M$ is finitely generated over $A$.

Proof. We first remark that $\operatorname{Tor}_{j}^{A}(M, k)=\operatorname{Tor}_{j}^{A[t]}(M, k[t])$. This follows from the fact that any free resolution of $k$ over $A$, tensored over $k$ with $k[t]$, gives a free resolution of $k[t]$ over $A[t]$.

The sequence (2.1) induces the exact sequence

$$
\cdots \rightarrow \operatorname{Tor}_{2}^{A[t]}(M, k) \rightarrow \operatorname{Tor}_{1}^{A[t]}(M, k[t]) \stackrel{\theta}{\longrightarrow} \operatorname{Tor}_{1}^{A[t]}(M, k[t]) \rightarrow \operatorname{Tor}_{1}^{A[t]}(M, k) \rightarrow \cdots .
$$

If $\operatorname{Tor}_{1}^{A[t]}(M, k)=0$, then the map $\theta$ is surjective. But it is also of positive degree, hence zero since all modules are bounded from below. Therefore, $\operatorname{Tor}_{1}^{A}(M, k)=$ $\operatorname{Tor}_{1}^{A[t]}(M, k[t])=0$.

If $\operatorname{Tor}_{2}^{A[t]}(M, k)=0$, then $\theta$ is injective. But $\operatorname{Tor}_{1}^{A[t]}(M, k[t])=\operatorname{Tor}_{1}^{A}(M, k)$ is a finitely generated $A$-module such that the $A$-action factors through the augmentation $A \rightarrow k$. Hence $\operatorname{Tor}_{1}^{A}(M, k)$ is a finitely generated $k$-module, which is necessarily bounded from above. Therefore, the map $\theta$, which raises degrees, cannot be injective unless $\operatorname{Tor}_{1}^{A}(M, k)$ vanishes.

The equivalence of conditions (iii) and (iv) of Theorem 1.1 is purely algebraic in nature and moreover well-known if $k$ is a field. The conditions are then equivalent to $M=H_{T}^{*}(X)$ being free over $A=H^{*}(B T)$. This does not hold in general, cf. Remark 2.4 and Example 5.2

Proposition 2.3. Let $M$ be an A-module. Then $\operatorname{Tor}_{1}^{A}(M, k)$ vanishes if and only if $\operatorname{Tor}_{j}^{A}(M, k)$ vanishes for all $j>0$.

Proof. We proceed by induction on the number $r$ of indeterminates of $A=k\left[t_{1}, \ldots, t_{r}\right]$. For $r \leq 1$ there is nothing to prove.

So assume that we have shown the equivalence for all $A$-modules, and let $M$ be an $A[t]$-module such that $\operatorname{Tor}_{1}^{A[t]}(M, k)=0$. Again, we take a portion of the long exact Tor sequence induced by the sequence (2.1),

$\cdots \rightarrow \operatorname{Tor}_{2}^{A}(M, k) \rightarrow \operatorname{Tor}_{2}^{A[t]}(M, k) \rightarrow \operatorname{Tor}_{1}^{A}(M, k) \rightarrow \operatorname{Tor}_{1}^{A}(M, k) \rightarrow \operatorname{Tor}_{1}^{A[t]}(M, k)=0$.

(Recall that $\operatorname{Tor}_{1}^{A[t]}(M, k[t])=\operatorname{Tor}_{1}^{A}(M, k)$.) As in the proof of Lemma 2.2 (i), we see that $\operatorname{Tor}_{1}^{A}(M, k)$ vanishes, hence by assumption $\operatorname{Tor}_{j}^{A}(M, k)$ for $j>0$. This implies that $\operatorname{Tor}_{j}^{A[t]}(M, k)$ vanishes as well for $j>0$. 
Remark 2.4. Since a free resolution of a $k$-module $N$ extends to a free resolution of the extended module $N \otimes A$, one gets $\operatorname{Tor}_{j}^{A}(N \otimes A, k)=0$ for $j>0$. (The converse does not hold as can be seen from Example [5.2)

More generally, consider the subalgebras $A^{\prime}=k\left[t_{1}, \ldots, t_{i}\right]$ and $A^{\prime \prime}=k\left[t_{i+1}, \ldots, t_{r}\right]$ of $A$. Let $M^{\prime}$ be an $A^{\prime}$-module. Since the Koszul resolution of $k$ over $A^{\prime}$ has length $i$, $\operatorname{Tor}_{j}^{A^{\prime}}\left(M^{\prime}, k\right)$ vanishes for $j>i$. Tensoring a free resolution of the $A^{\prime}$-module $M^{\prime}$ with $A^{\prime \prime}$ gives a free resolution of $M^{\prime} \otimes A^{\prime \prime}$ over $A=A^{\prime} \otimes A^{\prime \prime}$. By the Künneth theorem, this implies $\operatorname{Tor}_{j}^{A}\left(M^{\prime} \otimes A^{\prime \prime}, k\right)=\operatorname{Tor}_{j}^{A^{\prime}}\left(M^{\prime}, k\right) \otimes A^{\prime \prime}=0$ for $j>i$.

Lemma 2.5. Let $M$ be an A-module. If $\operatorname{Tor}_{1}^{A}(M, k)$ vanishes, then the localisation map $M \rightarrow S^{-1} M$ is injective for the multiplicative subset $S \subset A$ generated by the linear elements which can be extended to a basis of $A^{2}$.

Proof. Assume that the localisation map is not injective. Then there exist a nonzero $m \in M$ and a linear element $t \in S$ with $t m=0$. Consider again the exact sequence (2.1). Tensoring with $M$ over $k[t]$ gives a map $M \rightarrow M$ which sends $m^{\prime}$

to $t m^{\prime}$. This map is not injective because $t m=0$. Hence $\operatorname{Tor}_{1}^{k[t]}(M, k) \neq 0$. Since we may extend $t=t_{1}$ to a basis $\left(t_{1}, \ldots, t_{n}\right)$ of $A^{2}$, we get by Lemma 2.2 (1)

$$
\operatorname{Tor}_{1}^{k\left[t_{1}\right]}(M, k) \neq 0 \Rightarrow \operatorname{Tor}_{1}^{k\left[t_{1}, t_{2}\right]}(M, k) \neq 0 \Rightarrow \cdots \Rightarrow \operatorname{Tor}_{1}^{k\left[t_{1}, \ldots, t_{r}\right]}(M, k) \neq 0,
$$

which contradicts the assumption $\operatorname{Tor}_{1}^{A}(M, k)=0$.

\section{A NEW GROUND RING}

In the course of our proof we want to choose a common complement for a finite set of direct summands of the same rank in $k^{n}$. This is no problem if $k$ is $\mathbb{Q}$ or $\mathbb{R}$ (or any other infinite field), but it is not possible in general for $k=\mathbb{Z}$. Therefore, we extend the coefficients, a step which would be unnecessary in the case of an infinite field.

Note that in Theorem 1.1 we may replace $\mathbb{Z}$ by any $\mathbb{Z}$-algebra $k$ which is a flat and faithful $\mathbb{Z}$-module, i.e., such that the functor $-\otimes_{\mathbb{Z}} k$ is exact and faithful. In other words, $k$ is $\mathbb{Z}$-torsion-free, and for all $\mathbb{Z}$-modules $N$ one has $N \otimes_{\mathbb{Z}} k=0$ if and only if $N=0$. Indeed, for such a $k$ we have by the Universal Coefficient Theorem:

(1) $H^{*}\left(C \otimes_{\mathbb{Z}} k\right)=H^{*}(C) \otimes_{\mathbb{Z}} k$ for $C$, a complex,

(2) $\operatorname{Tor}^{H^{*}(B T ; k)}\left(M \otimes_{\mathbb{Z}} k, k\right)=\operatorname{Tor}^{H^{*}(B T ; \mathbb{Z})}(M, \mathbb{Z}) \otimes_{\mathbb{Z}} k$ for $M$, an $H^{*}(B T ; \mathbb{Z})$ module.

Hence all conditions of Theorem 1.1 hold over $\mathbb{Z}$ if and only if they hold over $k$.

We will use this to pass from $\mathbb{Z}$ to the localisation of $\mathbb{Z}\left[s_{1}, \ldots, s_{n}\right]$ with respect to all homogeneous elements which are $\mathbb{Z}$-reduced, i.e., which are not divisible by any integer $>1$. We will call this new (ungraded) algebra $k$. Since $\mathbb{Z}\left[s_{1}, \ldots, s_{n}\right]$ is factorial, no prime integer in $k$ is invertible. This implies in particular that $N \otimes_{\mathbb{Z}} k \neq 0$ for $N=\mathbb{Z} / m \mathbb{Z}$, hence for all $N \neq 0$. Because $k$ is $\mathbb{Z}$-torsion-free as well, it is flat and faithful over $\mathbb{Z}$.

Let $W_{n-i} \subset k^{n}$ be the submodule generated by

$$
w_{1}=\left(s_{1}, \ldots, s_{n}\right), w_{2}=\left(s_{1}^{2}, \ldots, s_{n}^{2}\right), \ldots, w_{i}=\left(s_{1}^{i}, \ldots, s_{n}^{i}\right) .
$$

It has the following nice property:

Lemma 3.1. Let $V \subset \mathbb{Z}^{n}$ a direct summand and denote the induced direct summand of $k^{n}$ by $V^{\prime}$.

(1) If $\operatorname{dim} V=i$, then $V^{\prime} \oplus W_{i}=k^{n}$.

(2) If $\operatorname{dim} V>i$, then $V^{\prime} \cap W_{i}$ contains an element which can be extended to a basis of $W_{i}$. 
Proof. (1) Choose a basis of $V$ and consider the matrix $U$ whose rows are these basis vectors. Then the greatest common divisor of all $i \times i$-minors of $U$ is 1 . Let $U^{\prime}$ be the $n \times n$-matrix obtained by appending the row vectors $w_{1}, \ldots, w_{n-i}$ to $U$. The determinant of $U^{\prime}$ is a $\mathbb{Z}$-reduced element of $\mathbb{Z}\left[s_{1}, \ldots, s_{n}\right]$, hence invertible in $k$. This proves the claim.

(2) We may assume $\operatorname{dim} V=i+1$. By part (11), we have $V^{\prime} \oplus W_{i+1}=k^{n}$. Therefore, $w_{n-i}$ can be written as the sum of some $v^{\prime} \in V^{\prime}$ and a linear combination $w$ of $w_{1}, \ldots, w_{n-i-1}$. Hence $v^{\prime}=w_{n-i}-w \in V^{\prime} \cap W_{i}$ can be extended to the basis $\left(w_{1}, \ldots, w_{n-i-1}, v^{\prime}\right)$ of $W_{i}$.

From now on to the end of the proof of Theorem 1.1 in Section $4 H^{*}(\cdot)$ denotes cohomology with coefficients in the new ground ring $k$. We set $A=H^{*}(B T)=$ $k\left[t_{1}, \ldots, t_{n}\right]$. The $k$-module $A^{2}$ is identified with $k^{n}$, using the basis $\left(t_{1}, \ldots, t_{n}\right)$. In particular, we consider each $W_{i}$ as contained in $A^{2}$.

We will need the following refined version of the Localisation Theorem.

Proposition 3.2. Let $X$ be a T-space as above with connected isotropy groups. Then the map

$$
H_{T}^{*}\left(X, X_{i-1}\right) \rightarrow H_{T}^{*}\left(X_{i}, X_{i-1}\right)
$$

becomes an isomorphism after localisation with respect to the multiplicative subset $S_{i}$ generated by all linear elements in $W_{i}$ which can be extended to a basis of $A^{2} \cong k^{n}$.

Proof. For $x \in X$ let $V_{x}$ be the kernel of the map $H^{2}(B T) \rightarrow H^{2}\left(B T_{x}\right)$, induced by the inclusion $T_{x} \hookrightarrow T$. Since $T_{x}$ is assumed to be connected, $V_{x}$ is a direct summand of $H^{2}(B T)$ with $\operatorname{dim} V_{x}+\operatorname{dim} T_{x}=n$. By a standard argument (induction over attaching equivariant cells to $X_{i}$ in order to get $X$, cf. tD. III.3.8] or AP 3.1.6]), it suffices to show that $S_{i}^{-1} H_{T}^{*}\left(T / T_{x}\right)=0$ for all $x \in X \backslash X_{i}$. Since $\operatorname{dim} V_{x}>i$ for such $x$, Lemma 3.1(2) gives us an $s \in S_{i} \cap V_{x}$ that annihilates $H_{T}^{*}\left(T / T_{x}\right)=H^{*}\left(B T_{x}\right)$.

Remark 3.3. It can be seen directly from the proof of Proposition 3.2 that, for a fixed $i=0, \ldots, n$, instead of demanding the connectedness of all isotropy groups it suffices that for $x \in X \backslash X_{i}$ the kernel of $H^{2}(B T) \rightarrow H^{2}\left(B T_{x}\right)$ contains a direct summand of dimension $>i$. This means that for all $x \in X \backslash X_{i}$ the isotropy group $T_{x}$ is contained in a subtorus of dimension $n-(i+1)$, e. g., for $i=0$ all isotropy groups different from $T$ should be contained in a proper subtorus.

Let $A_{i} \subset A$ be the subalgebra generated by $W_{i} \subset A^{2}$. Note that $A_{i}$ is noetherian because $k$ is.

Lemma 3.4. Suppose that all isotropy groups of $X$ are connected. Then the $A$ module $H_{T}^{*}\left(X, X_{i-1}\right)$, considered as $A_{i}$-module via restriction, is finitely generated.

Proof. Since $A_{i}$ is noetherian, it suffices (using long exact cohomology sequences) to show that, for each $x \in X \backslash X_{i-1}, H_{T}^{*}\left(T / T_{x}\right)=H^{*}\left(B T_{x}\right)$ is finitely generated over $A_{i}$. But since $T_{x}$ is assumed to be connected, $\operatorname{dim} V_{x} \geq i$ and $V_{x}+W_{i}=A^{2}$ for all $x \in X \backslash X_{i-1}$. So $H^{*}\left(B T_{x}\right)$ is a quotient of $A_{i}$ because the map $W_{i} \rightarrow$ $H^{2}(B T) \rightarrow H^{2}\left(B T_{x}\right)$ is surjective.

Remark 3.5. Again, for a fixed $i=0, \ldots, n$, one can see directly from the proof of Lemma 3.4 that it holds under a weaker condition than the connectedness of the isotropy groups. One needs that $V_{x}+W_{i}=A^{2}$ for all $x \in X \backslash X_{i-1}$. This holds if $T_{x}$ is contained in a subtorus of dimension $n-i$ for all $x \in X \backslash X_{i-1}$. 
Lemma 3.6. The following torsion products vanish:

(1) $\operatorname{Tor}_{j}^{A}\left(H_{T}^{*}\left(X_{i}, X_{i-1}\right), k\right)=0$ for $j>i$.

(2) $\operatorname{Tor}_{j}^{A_{i}}\left(H_{T}^{*}\left(X_{i}, X_{i-1}\right), k\right)=0$ for $j>0$.

Proof. The quotient $X_{i} / X_{i-1}$ is a bouquet of spaces, each of them fixed by a subtorus of codimension $i$. Let $Y$ be such a space, fixed by $T^{\prime \prime} \subset T$, and let $T^{\prime}$ be a torus complement to $T^{\prime \prime}$ in $T$. Since $H^{*}(B T)=H^{*}\left(B T^{\prime}\right) \otimes H^{*}\left(B T^{\prime \prime}\right)$ and $H_{T}^{*}(Y, \mathrm{pt})=H_{T^{\prime}}^{*}(Y, \mathrm{pt}) \otimes H^{*}\left(B T^{\prime \prime}\right)$, we are in the situation of Remark 2.4 with $A^{\prime}=H^{*}\left(B T^{\prime}\right), A^{\prime \prime}=H^{*}\left(B T^{\prime \prime}\right), M^{\prime}=H_{T^{\prime}}^{*}(Y, \mathrm{pt})$ and $M^{\prime} \otimes A^{\prime \prime}=H_{T}^{*}(Y, \mathrm{pt})$. Hence, $\operatorname{Tor}_{j}^{A}\left(H_{T}^{*}(Y, \mathrm{pt}), k\right)=0$ for $j>i$. Because $H_{T}^{*}\left(X_{i}, X_{i-1}\right)$ is a direct sum of terms of the form $H_{T}^{*}(Y, \mathrm{pt})$, this implies (1).

In order to prove (2), it suffices to show that each $H_{T}^{*}(Y, \mathrm{pt})=M^{\prime} \otimes A^{\prime \prime}$ is an extended $A_{i}$-module, again by Remark 2.4 One has $H^{2}(B T)=H^{2}\left(B T^{\prime}\right) \oplus W_{i}$ by Lemma 3.1], hence $A=A^{\prime} \otimes A_{i}$. Therefore, any $A^{\prime}$-equivariant map from $M^{\prime}$ to some $A$-module $N$ uniquely extends to an $A$-equivariant map $M^{\prime} \otimes A_{i} \rightarrow N$. This applies in particular to the canonical inclusion $M^{\prime} \rightarrow M^{\prime} \otimes A^{\prime \prime}$. Since we also have $A=A^{\prime} \otimes A^{\prime \prime}$, we can reverse the roles of $A_{i}$ and $A^{\prime \prime}$ to get a map in the other direction. By uniqueness, the compositions must be the identities on $M^{\prime} \otimes A_{i}$ and $M^{\prime} \otimes A^{\prime \prime}$, respectively. Hence, both $A$-modules are isomorphic.

\section{Proof of Theorem 1.1}

The equivalence (iii) $\Leftrightarrow$ iv is a special case of Proposition 2.3 We will show the implications (ii) $\Rightarrow$ (iii) $\Rightarrow$ (iii) $\Rightarrow$ (ii), (V) $\Rightarrow$ (iii) and, assuming that all isotropy groups are connected, (iv) $\Rightarrow(\underline{\nabla})$. The steps of the first chain of implications are either standard or quite easy. It is only in the most involved part of the proof, $(\mathrm{iv}) \Rightarrow(\mathbb{V})$, that we need the extension of the coefficients from $\mathbb{Z}$ to $k$. Recall that $A=H^{*}(B T)$ (with coefficients in $\left.k\right)$.

(ii) $\Rightarrow$ (ii): The surjectivity of $\iota^{*}$ implies that all boundaries starting at $E_{r}^{0, *}$ must vanish. Since $E_{2}^{p, q}=A^{p} \otimes H^{q}(X)$, the $A$-linearity of the boundary maps gives $d_{r}^{*, *}=0$ for all $r \geq 2$, i.e., $E_{2}=E_{\infty}$.

(iii) $\Rightarrow$ (iii): The abutment $E_{\infty}$ of the Serre spectral sequence can be considered as the (bi)graded $A$-module associated to a filtration of $H_{T}^{*}(X)$ by $A$-submodules $\mathcal{F}_{q}$ such that $E_{\infty}^{*, q}=\mathcal{F}_{q} / \mathcal{F}_{q-1}$, cf. Q bottom of p. 554]. Since $X$ is assumed to be a finite $T$-CW complex, this filtration is finite. For $E_{\infty}=E_{2}=A \otimes H^{*}(X)$ we have $\operatorname{Tor}_{j}^{A}\left(E_{\infty}^{*, q}, k\right)=0$ for $j>0$ and any $q$ (see Remark 2.4). Hence, via the long exact Tor sequences coming from the short exact sequences $0 \rightarrow \mathcal{F}_{q-1} \rightarrow \mathcal{F}_{q} \rightarrow E_{\infty}^{*, q} \rightarrow 0$ one gets by induction that $\operatorname{Tor}_{j}^{A}\left(H_{T}^{*}(X), k\right)=0$ for $j>0$.

(iii) $\Rightarrow$ (i): This follows immediately from the Eilenberg-Moore spectral sequence $\operatorname{Tor}_{*}^{A}\left(H_{T}^{*}(X), k\right) \Rightarrow H^{*}(X)$. A version of the Eilenberg-Moore theorem suitable for our purpose can be found in GM, Corollary 3.5].

Before entering the central part of our proof, we reformulate condition (v).

Lemma 4.1. The Atiyah-Bredon sequence is exact if and only if for all $0 \leq i \leq n$ the long exact sequence (1.1) splits into short exact sequences

$$
0 \longrightarrow H_{T}^{*}\left(X, X_{i-1}\right) \stackrel{\alpha_{i}}{\longrightarrow} H_{T}^{*}\left(X_{i}, X_{i-1}\right) \stackrel{\delta_{i}}{\longrightarrow} H_{T}^{*+1}\left(X, X_{i}\right) \longrightarrow 0
$$


Proof. The idea is to combine the Atiyah-Bredon sequence as follows with the short sequences (4.1) to a commutative diagram:

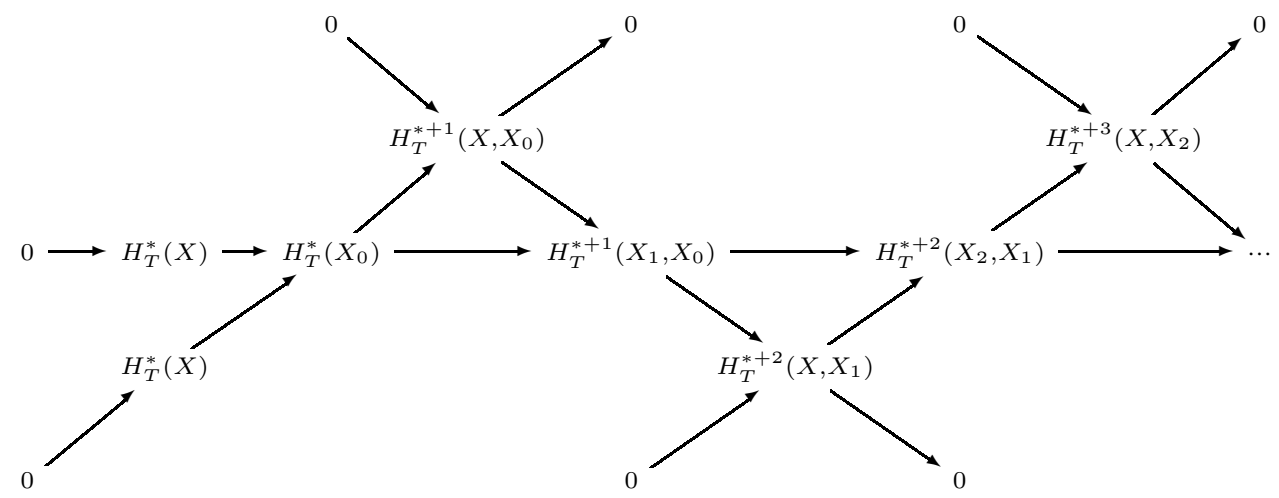

Assume that the Atiyah-Bredon sequence is exact. Using that the diagonal sequences come from the long exact sequence (1.1), one shows inductively that they are in fact short exact. The reverse direction is done by diagram chase.

(vi) $\Rightarrow$ (iii): By downward induction on $i$, we prove the statement

$$
\operatorname{Tor}_{j}^{A}\left(H_{T}^{*}\left(X, X_{i-1}\right), k\right)=0 \text { for all } j>i .
$$

For $i=n+1$ there is nothing to prove. The induction step follows from Lemma 3.6] (1) and the long exact Tor sequence applied to the short exact sequence (4.1). Since $X_{-1}=\emptyset$, the case $i=0$ is condition (iii).

(iv) $\Rightarrow(\underline{\nabla})$ : Here we assume all isotropy groups to be connected. We will show that for $0 \leq i \leq n$ the condition

$$
\operatorname{Tor}_{1}^{A_{i}}\left(H_{T}^{*}\left(X, X_{i-1}\right), k\right)=0
$$

(which is satisfied for $i=0$ by hypothesis) implies that the sequence (4.1) is exact and that (4.3) holds for $i+1$ instead of $i$. This would prove the claim by induction.

Suppose that (4.3) is true. By Lemma 2.5 the localisation map $H_{T}^{*}\left(X, X_{i-1}\right) \rightarrow$ $S_{i}^{-1} H_{T}^{*}\left(X, X_{i-1}\right)$ is injective. Moreover, Proposition 3.2 implies that the localised map $S_{i}^{-1} \alpha_{i}$ is an isomorphism. Hence $\alpha_{i}$ is injective, and (4.1) is exact.

Applying $\operatorname{Tor}_{*}^{A_{i}}(-, k)$ to (4.1) gives the exact sequence

$$
\operatorname{Tor}_{2}^{A_{i}}\left(H_{T}^{*}\left(X_{i}, X_{i-1}\right), k\right) \longrightarrow \operatorname{Tor}_{2}^{A_{i}}\left(H_{T}^{*}\left(X, X_{i}\right), k\right) \longrightarrow \operatorname{Tor}_{1}^{A_{i}}\left(H_{T}^{*}\left(X, X_{i-1}\right), k\right) .
$$

The first term vanishes by Lemma 3.6 (2) and the third one by hypothesis. Therefore, $\operatorname{Tor}_{2}^{A_{i}}\left(H_{T}^{*}\left(X, X_{i}\right), k\right)$ vanishes as well, as does $\operatorname{Tor}_{1}^{A_{i+1}}\left(H_{T}^{*}\left(X, X_{i}\right), k\right)$ by Lemmas 3.4 and 2.2(ii) since $A_{i+1}=A_{i}[t]$ for some $t \in A^{2}$. This finishes the proof of Theorem 1.1

Remark 4.2. Using Remarks 3.3 and 3.5 we get a partial result by the same reasoning as above if instead of demanding the connectedness of all isotropy groups, we make the following weaker assumptions for some $i_{0}<n$ :

(1) $T_{x}$ is connected for all $x \in X_{i_{0}+1}$,

(2) $T_{x}$ is contained in a subtorus of dimension $n-\left(i_{0}+1\right)$ for $x \notin X_{i_{0}+1}$.

Condition (iv) then implies that the sequences (4.1) are exact for $i=0, \ldots, i_{0}$. This means that we get an exact sequence

$$
0 \longrightarrow H_{T}^{*}(X) \longrightarrow H_{T}^{*}\left(X_{0}\right) \longrightarrow H_{T}^{*+1}\left(X_{1}, X_{0}\right) \longrightarrow \cdots \longrightarrow H_{T}^{*+i_{0}}\left(X_{i_{0}}, X_{i_{0}-1}\right) .
$$

\section{EXAMPLES}

Here we use integer coefficients again. As in Remark 2.4 we call an $H^{*}(B T)$-module extended if it is isomorphic to $H^{*}(B T) \otimes_{\mathbb{Z}} N$ for some $\mathbb{Z}$-module $N$. 


\subsection{Hamiltonian $T$-manifolds.}

Theorem 5.1. Let $X$ be a compact Hamiltonian T-manifold with connected isotropy groups. Then $H_{T}^{*}(X)$ is an extended $H^{*}(B T)$-module.

More precisely, if $F_{1}, \ldots, F_{s}$ are the (finitely many) components of $X^{T}$, then there are even natural numbers $\lambda_{1}, \ldots, \lambda_{s}$ such that

$$
H_{T}^{*}(X)=\bigoplus_{k} H^{*}(B T) \otimes H^{*-\lambda_{k}}\left(F_{k}\right) .
$$

The real version of this result (which does not need the connectivity assumption, cf. Remark 1.2) goes back to Frankel [F], but see also Atiyah-Bott [AB, Kirwan $\mathrm{K}$ and (for the method of proof used below) Tolman-Weitsman $\mathrm{TW}_{2}$. Note that our result also works if the cohomology of the fixed point components has torsion.

Proof. Let $\mu: X \rightarrow \mathfrak{t}^{*}$ be a moment map for the $T$-action on $X$. For generic $\xi \in \mathfrak{t}$ the function $f: x \mapsto\langle\mu(x), \xi\rangle$ is an equivariant Morse-Bott function on $X$ whose critical set is exactly $X^{T}$. We may assume that the critical values $c_{k}=f\left(F_{k}\right)$ are distinct. We will prove by induction on $k$ that $H_{T}^{*}\left(X_{k}^{+}\right)$is a finitely generated extended $H^{*}(B T)$-module, where $X_{k}^{+}=f^{-1}\left(\left(-\infty, c_{k}+\varepsilon\right]\right)$ with $\varepsilon$ so small that the interval $\left[c_{k}-\varepsilon, c_{k}+\varepsilon\right]$ contains no critical value other than $c_{k}$.

In addition to $X^{+}=X_{k}^{+}$introduce $X^{-}=f^{-1}\left(\left(-\infty, c_{k}-\varepsilon\right]\right)$, the negative disc bundle $D$ and the negative sphere bundle $S$ to $F=F_{k}$. Since the pair $\left(X^{+}, X^{-}\right)$ can be retracted to $(D, S)$, we have

$$
H_{T}^{*}\left(X^{+}, X^{-}\right)=H_{T}^{*}(D, S)=H_{T}^{*-\lambda}(F)
$$

by the Thom isomorphism, where $\lambda$ is the (even) Morse index of $F$. The composition

$$
H_{T}^{*-\lambda}(F)=H_{T}^{*}\left(X^{+}, X^{-}\right) \rightarrow H_{T}^{*}\left(X^{+}\right) \rightarrow H_{T}^{*}(F)
$$

is multiplication by the equivariant Euler class $e_{D}$ of the bundle $D \rightarrow F$. The latter becomes, after restriction to any point $x \in F$, the product of the non-zero weights of the representation of $T$ on the tangent space to $D$ at $x$. In particular, $e_{D}$ is not divisible by any prime integer, so that multiplication by $e_{D}$ is injective and does not change divisibility by prime integers.

By injectivity, the long exact sequence for the pair $\left(X^{+}, X^{-}\right)$splits into short exact sequences

$$
0 \longrightarrow H_{T}^{*}\left(X^{+}, X^{-}\right) \longrightarrow H_{T}^{*}\left(X^{+}\right) \longrightarrow H_{T}^{*}\left(X^{-}\right) \longrightarrow 0 .
$$

We claim that this sequence is split. This will imply our claim that $H_{T}^{*}\left(X^{+}\right)$is a finitely generated extended $H^{*}(B T)$-module.

By induction, $H_{T}^{*}\left(X^{-}\right)$is extended, say of the form $H^{*}(B T) \otimes N$, where $N$ is the direct sum of some $H^{*}\left(F_{i}\right)$, with degree shifts. In particular, $N$ is a finitely generated $\mathbb{Z}$-module. A section of $H^{*}(B T)$-modules to $H_{T}^{*}\left(X^{+}\right) \rightarrow H_{T}^{*}\left(X^{-}\right)$is the same as a section of $\mathbb{Z}$-modules $N \rightarrow H_{T}^{*}\left(X^{+}\right)$. The latter exists if and only if every element of $N$ has a preimage with the same annihilator. Take a torsion element $\gamma \in N$, whose annihilator is generated by some $m \in \mathbb{Z}$. Let $\beta \in H_{T}^{*}\left(X^{+}\right)$ be a preimage of $\gamma$. Then $m \beta$ is in the image of some $\alpha \in H_{T}^{*}\left(X^{+}, X^{-}\right)$. Since $m \beta$ is divisible by $m$, so is $e_{D} \alpha$, hence also $\alpha$. We finally subtract the image of $\alpha / m$ from $\beta$ to get the preimage of $\gamma$ we were looking for.

Together with Theorem 1.1 this Theorem gives the injectivity result of TolmanWeitsman $\mathrm{TW}_{1}$ Proposition 7.2] and Schmid's version of the Chang-Skjelbred lemma [S] Théorème 3.2.1] in the case of connected isotropy groups. For a discussion of non-connected isotropy groups see [FP]; cf. also $\left[\mathrm{TW}_{2}\right.$, Section 4]. 
5.2. Other examples and counterexamples. We saw in Remark 2.4 that a $T$-space satisfies condition (iii) of Theorem 1.1 if its equivariant cohomology is extended. The converse does not hold in general.

Example 5.2. Here we take $T=S^{1}$. Let $X=S^{2} \cup_{\phi} C \mathbb{R} \mathbb{P}^{2} \times S^{1}$, where $C \mathbb{R P}^{2}$ is the cone over $\mathbb{R P}^{2}$ and $\phi: \mathbb{R P}^{2} \times S^{1} \stackrel{\text { pr }}{\longrightarrow} \mathbb{R P}^{2} \rightarrow \mathbb{R P ^ { 2 }} / \mathbb{R P}^{1}=S^{2}$. The $S^{1}$-action is trivial on $S^{2}$ and free on $C \mathbb{R P}^{2} \times S^{1}$ (acting on the second component), so all isotropy groups are connected. It is easy to calculate the (equivariant) cohomology of $X$ using the Mayer-Vietoris sequence. One obtains:

$$
\begin{aligned}
& H^{i}(X)= \begin{cases}\mathbb{Z} & i=0,2, \\
\mathbb{Z} / 2 \mathbb{Z} & i=4, \\
0 & \text { otherwise, }\end{cases} \\
& H_{T}^{*}(X) \cong \mathbb{Z}[t] \oplus \operatorname{ker}(\mathbb{Z}[t] \rightarrow \mathbb{Z} / 2 \mathbb{Z})[+2],
\end{aligned}
$$

where the map $\mathbb{Z}[t] \rightarrow \mathbb{Z} / 2 \mathbb{Z}$ is the composition of the augmentation with the projection $\mathbb{Z} \rightarrow \mathbb{Z} / 2 \mathbb{Z}$ and "[+2]" denotes a degree shift by +2 .

The equivalent conditions (1) to (V) are satisfied in this example, but $H_{T}^{*}(X)$ is not extended, in particular, $\iota^{*}: H_{T}^{*}(X) \rightarrow H^{*}(X)$ does not have a section.

In order to get exactness of the Atiyah-Bredon sequence, we assumed that all isotropy groups are connected. This requirement cannot be dropped in general.

Example 5.3. Let $X=\mathbb{R P}^{2}$, considered as the quotient of the disk $D^{2}$ by identifying opposite points on the boundary $S^{1}$. The standard rotation of $T=S^{1}$ on $D^{2}$ descends to $\mathbb{R P}^{2}$. Note that the points on $\mathbb{R P}^{1}=S^{1} /\{ \pm 1\}$ have $\mathbb{Z}_{2}$ as isotropy group.

The open sets $\mathbb{R P}^{2} \backslash \mathbb{R P}^{1}$ and $\mathbb{R P}^{2} \backslash 0$ cover $\mathbb{R P}^{2}$. The Mayer-Vietoris sequence for the equivariant cohomology of $\mathbb{R P}^{2}$ splits into short exact sequences, which gives

$$
H_{T}\left(\mathbb{R} \mathbb{P}^{2}\right)=\mathbb{Z}[t] \oplus \mathbb{Z} / 2 \mathbb{Z}[t][+2]=H^{*}\left(\mathbb{R}^{2}\right) \otimes H^{*}(B T)
$$

Hence, $H_{T}^{*}\left(\mathbb{R P}^{2}\right)$ is an extended $H^{*}(B T)$-module, but the map $H_{T}^{*}(X) \rightarrow H_{T}^{*}\left(X^{T}\right)$ cannot be injective because $H_{T}^{*}\left(X^{T}\right)=H_{T}^{*}(\mathrm{pt})$ is free over $\mathbb{Z}$.

By taking products of $\mathbb{R P}^{2}$ with itself, one obtains analogous examples for higherdimensional $T$.

The situation is different if one assumes $H_{T}^{*}(X)$ to be free over $H^{*}(B T)$. The main result of $[\mathrm{FP}]$ is that in this case one can allow isotropy groups with at most one (finite) cyclic factor and still get the exact Atiyah-Bredon sequence. An example due to Tolman-Weitsman $\mathrm{TW}_{2}$, Section 4], $S^{2} \times S^{2}$ with double speed rotation on each factor, shows that isotropy groups with two cyclic factors are not allowed.

It is not difficult to show that if $\operatorname{Tor}_{1}^{H^{*}(B T)}\left(H_{T}^{*}(X), \mathbb{Z}\right)=0$ and $H_{T}^{*}(X)$ is free over $\mathbb{Z}$, then the map $H_{T}^{*}(X) \rightarrow H_{T}^{*}\left(X^{T}\right)$ is always injective, no matter what isotropy groups occur. But these assumptions do not guarantee exactness of the Atiyah-Bredon sequence at $H_{T}^{*}\left(X^{T}\right)$ in the presence of non-connected isotropy groups.

Example 5.4. Starting with the $S^{1}$-space $X$ from Example 5.2 we consider the space $Y=X \times S^{2}$ with the componentwise action of $T=S^{1} \times S^{1}$, where $S^{1}$ acts on $S^{2}$ with double speed rotation. Then $H_{T}^{*}(Y)=H_{S^{1}}(X) \otimes H_{S^{1}}\left(S^{2}\right)$ is not free over $H^{*}(B T)$. But since $H_{S^{1}}(X)$ and $H_{S^{1}}\left(S^{2}\right)$ are both free over $\mathbb{Z}$, it is easy to check that $H_{T}^{*}(Y) \rightarrow H_{T}^{*}\left(Y_{0}\right)$ is injective. Because $H_{T}^{*}\left(Y, Y_{0}\right)$ contains an element of order 4 , while $H_{T}^{*}\left(Y_{1}, Y_{0}\right)$ does not, $H_{T}^{*}\left(Y, Y_{0}\right) \rightarrow H_{T}^{*}\left(Y_{1}, Y_{0}\right)$ cannot be injective. In other words, the sequence $H_{T}^{*}(Y) \rightarrow H_{T}^{*}\left(Y_{0}\right) \rightarrow H_{T}^{*+1}\left(Y_{1}, Y_{0}\right)$ is not exact.

Clearly, for $T=S^{1}$ the Atiyah-Bredon sequence is exact if and only if the restriction map $H_{T}^{*}(X) \rightarrow H_{T}^{*}\left(X_{0}\right)$ is injective. Our last example shows that this 
equivalence is false for higher-dimensional $T$, even if all isotropy groups are connected.

Example 5.5 (C. Allday). Let $X=\Sigma T$ be the suspension of $T$ with the standard level-wise action. Again the Mayer-Vietoris sequence gives the equivariant cohomology:

$$
\begin{aligned}
& \tilde{H}^{*}(X) \cong \tilde{H}^{*}(T)[+1], \\
& H_{T}^{*}(X) \cong \operatorname{ker}\left(\mathbb{Z}\left[t_{1}, \ldots, t_{n}\right] \oplus \mathbb{Z}\left[t_{1}, \ldots, t_{n}\right] \stackrel{(\varepsilon, \varepsilon)}{\longrightarrow} \mathbb{Z}\right),
\end{aligned}
$$

where $\varepsilon: \mathbb{Z}\left[t_{1}, \ldots, t_{n}\right] \rightarrow \mathbb{Z}$ denotes the augmentation. Note that only for $n=1$ we have that $H_{T}^{*}(X)$ is free over $H^{*}(B T)$. The map $H_{T}^{*}(X) \rightarrow H_{T}^{*}\left(X_{0}\right)$ clearly is injective, but for $n>1$ the Atiyah-Bredon sequence is exact only at $H_{T}^{*}(X)$.

\section{REFERENCES}

[AP] C. Allday and V. Puppe, Cohomological methods in transformation groups. Cambridge Stud. Adv. Math. 32, Cambridge Univ. Press, Cambridge 1993.

[A] M. F. Atiyah, Elliptic operators and compact groups, LNM 401, Springer, Berlin 1974.

[AB] M. F. Atiyah and R. Bott, The moment map and equivariant cohomology, Topology 23 (1984), 1-28.

[BBFK] G. Barthel, J.-P. Brasselet, K.-H. Fieseler, and L. Kaup, Combinatorial intersection cohomology for fans, Tohoku Math. J. 54 (2002), 1-41.

[B] G. E. Bredon, The free part of a torus action and related numerical equalities, Duke Math. J. 41 (1974), 843-854.

[CS] T. Chang and T. Skjelbred, The topological Schur lemma and related results, Ann. Math. 100 (1974), 307-321.

[tD] T. tom Dieck, Transformation groups, de Gruyter Stud. Math. 8, de Gruyter 1987.

$[\mathrm{F}] \quad$ T. Frankel, Fixed points and torsion on Kähler manifolds, Ann. Math., II. Ser. 70 (1959), $1-8$.

[FP] M. Franz and V. Puppe. Exact sequences for equivariant formal spaces, ArXiv e-print math.AT/0307112

[GKM] M. Goresky, R. Kottwitz and R. MacPherson. Equivariant cohomology, Koszul duality, and the localization theorem, Invent. Math. 131 (1998), 25-83.

[GM] V.K. A.M. Gugenheim and J.P. May, On the theory and applications of differential torsion products, Mem. Am. Math. Soc. 142 (1974).

[K] F. Kirwan, Cohomology of quotients in symplectic and algebraic geometry, Math. Notes 31. Princeton Univ. Press, Princeton, NJ 1984.

[Q] D. Quillen, The spectrum of an equivariant cohomology ring, I, Ann. Math. 94 (1971), 549-572.

[S] C. Schmid, Cohomologie équivariante de certaines variétés hamiltoniennes et de leur partie réelle, Ph.D. Thesis, Université de Genève 2001, available at http://www.unige.ch/math/biblio/these/theses.html.

$\left[\mathrm{TW}_{1}\right]$ S. Tolman and J. Weitsman, The cohomology rings of symplectic quotients, Comm. Anal. Geom. 11 (2003), 751-773.

$\left[\mathrm{TW}_{2}\right]$ S. Tolman and J. Weitsman. On the cohomology rings of Hamiltonian T-spaces, in Northern California symplectic geometry seminar, Transl., Ser. 2196 (45), Am. Math. Soc., Providence, R.I., 1999, 251-258.

Fachbereich Mathematik, Universität Konstanz, 78457 Konstanz, Germany

E-mail address: matthias.franz@ujf-grenoble.fr

E-mail address: volker.puppe@uni-konstanz.de 